\title{
Intestinal Cholesterol Absorption Efficiency in Man Is Related to Apoprotein E Phenotype
}

\author{
Y. Antero Kesäniemi, Christian Ehnholm, * and Tatu A. Miettinen \\ Second Department of Medicine, University of Helsinki, and *National Public Health Institute, Helsinki, Finland
}

\begin{abstract}
Relationship between the efficiency of cholesterol absorption and apolipoprotein $\mathbf{E}$ (apoE) phenotype was studied in a random sample of middle-aged Finnish men. Subjects that were either heterozygous or homozygous for the allele $\epsilon 2$ absorbed less and synthesized more cholesterol than those with the phenotype $E 4 / 3$ and $E 4 / 4$, the values for the individuals with the most frequent phenotype E3/3 (56\% of the population sample) falling in between. Among the whole study group, the sum of the subscripts of apoE phenotype (e.g., E2/3 = 5) was correlated positively with the fractional absorption of cholesterol $(r=0.40$; $P<0.05)$ and negatively with the serum level of lathosterol, a cholesterol precursor sterol reflecting the activity of cholesterol synthesis $(r=-0.48 ; P<0.01)$. Thus, apoE polymorphism appears to affect the efficiency of cholesterol absorption and may by this mechanism contribute to the variation in plasma total and low density lipoprotein cholesterol concentration.
\end{abstract}

\section{Introduction}

Several population studies have indicated that apolipoprotein $\mathrm{E}$ $(\text { apoE })^{1}$ polymorphism influences plasma lipid levels (1-5). Thus, subjects with the most frequent apoE phenotype E3/3 have lower plasma total and low density lipoprotein (LDL) cholesterol levels than those with E4/3 and E4/4 phenotypes, but higher levels than subjects with heterozygosity and particularly homozygosity for the $\epsilon 2$ allele. Recent studies have also shown that the variation in plasma total and LDL cholesterol concentration within the population is clearly determined by both LDL catabolism and synthesis (6), which, in turn, at least among the Finns, are regulated by the efficiency of cholesterol absorption (7). Thus, subjects with plasma cholesterol values in the highest decile have a higher fractional cholesterol absorption than those with the cholesterol values in the lowest decile (7). The occurrence of both

Dr. Kesäniemi is presently at the Department of Medicine, University of Oulu, Kajaanintie 50, SF-90220 Oulu, Finland. Address correspondence and reprint requests to Dr. Miettinen, Second Department of Medicine, University of Helsinki, Haartmaninkatu 4 SF-00290, Helsinki, Finland.

Received for publication 23 December 1986 and in revised form 23 April 1987.

1. Abbreviation used in this paper: apo, apolipoprotein.

J. Clin. Invest.

(c) The American Society for Clinical Investigation, Inc. $0021-9738 / 87 / 08 / 0578 / 04 \$ 2.00$

Volume 80, August 1987, 578-581 effective cholesterol absorption and increased $\epsilon 4$ allele frequency among the subjects with high plasma cholesterol levels prompted us to consider that apoE polymorphism influences intestinal absorption of cholesterol and consequently leads to variation in plasma cholesterol concentration. This hypothesis was tested by relating the efficiency of cholesterol absorption to the apoE phenotype in a random sample of Finnish men, a population characterized by a unique distribution of apoE alleles (3), a high serum cholesterol concentration, and a high incidence of coronary heart disease (8).

\section{Methods}

Subjects. A random sample of 300 men aged $35-50$ yr were identified from a population register in Helsinki. A fasting serum sample was obtained and analyzed for plasma lipids and lipoproteins. The limits for the lowest, modal, and highest deciles of LDL cholesterol were defined, and 13 subjects from each decile were recalled for the detailed analysis. The study population did not include any subjects with tendon xanthomata, which indicates that patients with familial hypercholesterolemia were most likely absent. No attempts were made to identify or exclude the presence of other types of primary hyperlipidemia. All the subjects volunteered for the studies, which had been approved by the Ethical Committee of our hospital.

Diet. All the subjects were studied while on their regular home diet. They were advised to continue their habitual diet and to keep a food record for $7 \mathrm{~d}$. From this the consumption of dietary constituents was calculated (9).

Laboratory procedures. Plasma lipids and lipoproteins were analyzed according to the Lipid Research Clinics Manual of Laboratory Operation (10). ApoE phenotyping was performed by isoelectric focusing (3). Cholesterol absorption was measured by the peroral double-isotope, continuous cholesterol-betasitosterol feeding method (11). For this purpose the subjects received a mixture of $4\left[{ }^{14} \mathrm{C}\right]$ cholesterol $(0.11 \mu \mathrm{Ci})$, $22,23\left[{ }^{3} \mathrm{H}\right]$ betasitosterol $(0.14 \mu \mathrm{Ci})$, and $\mathrm{Cr}_{2} \mathrm{O}_{3}(200 \mathrm{mg})$ three times a day with major meals for $7 \mathrm{~d}$ during dietary recording. At the end of the 7-d period, a 3-d stool collection was performed. Fecal outputs of bile acids and neutral sterols were determined with gas-liquid chromatography $(12,13)$ using a capillary column $(14)$, and overall cholesterol synthesis was calculated as the difference between fecal elimination of cholesterol as bile acids and neutral sterols and dietary cholesterol. The absorption and synthesis of cholesterol were assessed independently by the measurements (15) of serum campesterol (plant sterol), another indicator of cholesterol absorption (16), and serum lathosterol, a cholesterol precursor sterol shown to be a sensitive indicator of the activity of cholesterol synthesis $(17,18)$.

Statistical analysis. Student's $t$ test or analysis of variance was employed to compare the variables in the lipid and lipoprotein metabolism of the various groups of patients as indicated in Table I or Fig. 1. For the triglyceride analysis, the observed $\log$-normal distribution of the values was taken into account. Correlation coefficients were calculated by the least square method. The results are presented as means ( \pm SEM) and are consideréd to be significant if $P<0.05$. 


\section{Results}

The distribution of apoE phenotypes among the subjects studied is shown in Table I. Of the 39 subjects $56 \%$ had the phenotype $\mathrm{E} 3 / 3$; $33 \%$, the phenotype $\mathrm{E} 3 / 4$ or $\mathrm{E} 4 / 4$; and $10 \%$ were heterozygous or homozygous for the allele $\epsilon 2$. The distribution roughly corresponds to that found in the Finnish population (3). To relate cholesterol absorption to apoprotein $\mathrm{E}$ isoforms, the subjects were divided into three groups: first, persons either homozygous or heterozygous for the $\epsilon 2$ allele, designated as $\mathrm{E}_{2}$ subjects $(n=4)$; second, those with E3/3 $(n=22)$; and third, subjects with the phenotype E4/4 or E3/4 $(n=13)$, designated as $\mathrm{E}_{4}$ subjects.

The mean plasma total cholesterol concentration was lower in the subjects with E2 $(4.7 \pm 0.3[ \pm \mathrm{SE}] \mathrm{mmol} / \mathrm{liter})$ than in those with the phenotype E3/3 $(6.1 \pm 0.3 \mathrm{mmol} / \mathrm{liter})$ or in the E4 patients $(6.9 \pm 0.3 \mathrm{mmol} / \mathrm{liter})$. Also, the LDL cholesterol level in the E3/3 group was higher than that in the E2/2-4 subjects, but insignificantly lower than that in the patients with E4/4-3. Age, body-mass index (weight/height ${ }^{2}$ ), and other lipid values were quite similar in the three groups. However, the E2 and E4 homozygotes had somewhat elevated very low density lipoprotein (VLDL) cholesterol and total triglyceride levels. The dietary intakes of cholesterol in terms of milligrams/day per kilogram were not significantly different in the E2 $(6.4 \pm 0.5), E 3 / 3$ $(5.5 \pm 0.5)$, and E4 subjects $(6.5 \pm 0.6)$ (analysis of variance, $F$ value of 1.67). Also, the ratio of polyunsaturated to saturated fatty acids in dietary fat was similar in the three groups $(0.34 \pm 0.05,0.31 \pm 0.04$, and $0.28 \pm 0.04$, respectively) (analysis of variance; $F$ value of 1.27).

The homozygous E3/3 subjects had a higher percent absorption of cholesterol than the patients with E2 $(P<0.05)$; the E4 subjects having the highest values (Fig. 1). In fact, a positive correlation was observed between the percentage absorption of cholesterol and the sum of the subscripts of apoE (e.g., E2/3 $=5)(r=0.40 ; P<0.05)$ among all the subjects. The serum content of campesterol, another indicator of cholesterol absorption (16), was only insignificantly lower in the subjects with E2 $(162 \pm 14 \mu \mathrm{mol} / \mathrm{mmol}$ of cholesterol) than in those with E3/3 $(171 \pm 12)$ or $\mathrm{E} 4(197 \pm 30)$.
The findings for bile acid and cholesterol synthesis were opposite to those found for cholesterol absorption. Thus, the subjects with E4 tended to have lower bile acid and cholesterol synthesis than those with E3/3 and particularly E2 (Fig. 1). These differences did not reach statistical significance, but the serum content of a cholesterol precursor sterol, lathosterol, a sensitive indicator of the activity of cholesterol synthesis $(17,18)$, was significantly lower in the subjects with E4 than in those with E3/3 or E2 $(P<0.05)$ (Fig. 1). Also, the serum content of lathosterol was negatively correlated with the sum of the subscripts of apoE ( $r=-0.48 ; P<0.01)$ among all the subjects. Finally, the ratio of serum lathosterol to campesterol, which takes into account both the activity of cholesterol synthesis and the efficiency of cholesterol absorption was higher in the subjects with E2 $(1.13 \pm 0.03)$ than in those with E4 $(0.77 \pm 0.10)(P<0.01)$, the mean values for the subjects with E3/3 (1.08 \pm 0.14$)$ falling in between (analysis of variance; $F$ value of 3.22 ).

\section{Discussion}

The genetic polymorphism of apoE is controlled by three alleles, $\epsilon 2, \epsilon 3$, and $\epsilon 4$, at a single gene locus $(19,20)$. The amino acid substitutions in the main isoproteins have been characterized, and these differences change the affinity of apoE-containing lipoproteins for lipoprotein receptors, so that the affinity of apoE2 is lower than that of E3 and E4 (21-23). Another important aspect of the apoE polymorphism is the effects of its alleles on serum cholesterol levels. Thus, in several populations, including the Finns, the $\epsilon 2$ allele is associated with low and the $\epsilon 4$ allele, with high plasma total cholesterol, LDL cholesterol, and apoB levels (1-4). Recent reports have also indicated that the metabolism of triglyceride-rich lipoproteins and the kinetics of plasma apoE itself are markedly influenced by the apoE phenotype (2426), the plasma apoE concentration being lower in a homozygote for E4 than E3 (26). The present study demonstrates that variations at the apoE locus may also affect the efficiency of intestinal cholesterol absorption. All these metabolic events may be explained by a single mechanism, i.e., different recognition of apoE isoforms by cell receptors for apoE-containing lipoproteins.

Table I. Clinical Data and Plasma Lipids and Lipoproteins

\begin{tabular}{|c|c|c|c|c|c|c|c|c|c|}
\hline \multirow[b]{2}{*}{ Phenotype } & \multirow[b]{2}{*}{ No. } & \multirow[b]{2}{*}{ Percentage } & \multirow[b]{2}{*}{ Age } & \multirow[b]{2}{*}{ Weight/height ${ }^{2}$} & \multicolumn{4}{|l|}{ Cholesterol } & \multirow{2}{*}{$\begin{array}{l}\text { Total } \\
\text { triglycerides }\end{array}$} \\
\hline & & & & & Total & VLDL & LDL & HDL & \\
\hline & & $\%$ & $y r$ & & $\mathrm{mmol} / \mathrm{liter}$ & $\mathrm{mmol} / \mathrm{liter}$ & mmol/liter & $\mathrm{mmol} / \mathrm{liter}$ & \\
\hline $\mathrm{E} 2 / 2$ & 1 & 2.6 & 41 & 25.6 & 4.5 & 1.0 & 2.0 & 1.0 & 2.7 \\
\hline \multirow[t]{2}{*}{$\mathrm{E} 2 / 3$} & 2 & 5.1 & 39 & 23.6 & 4.2 & 0.29 & 2.4 & 1.4 & 0.8 \\
\hline & & & 37 & 25.8 & 4.4 & 0.84 & 2.5 & 1.3 & 1.1 \\
\hline $\mathrm{E} 2 / 4$ & 1 & 2.6 & 50 & 26.8 & 5.6 & 0.35 & 3.3 & 1.6 & 0.9 \\
\hline E3/3 & 22 & 56.4 & $45.5 \pm 1.1^{*}$ & $27.7 \pm 0.8$ & $6.1 \pm 0.3$ & $0.39 \pm 0.06$ & $4.2 \pm 0.2$ & $1.4 \pm 0.1$ & $1.3 \pm 0.2$ \\
\hline $\mathrm{E} 3 / 4$ & 12 & 30.8 & $46.3 \pm 1.4$ & $25.9 \pm 1.0$ & $6.7 \pm 0.2^{\ddagger}$ & $0.46 \pm 0.06$ & $4.8 \pm 0.2^{\ddagger 8}$ & $1.3 \pm 0.1$ & $1.5 \pm 0.1$ \\
\hline E4/4 & 1 & 2.6 & 50 & 25.4 & 9.9 & 0.80 & 7.1 & 2.0 & 2.5 \\
\hline E2/2-4" & 4 & 10.3 & $41.8 \pm 2.9$ & $25.5 \pm 0.7$ & $4.7 \pm 0.3$ & $0.63 \pm 0.18$ & $2.6 \pm 0.3$ & $1.3 \pm 0.1$ & $1.4 \pm 0.4$ \\
\hline $\mathrm{E} 3 / 3$ & 22 & 56.4 & $45.5 \pm 1.1$ & $27.7 \pm 0.8$ & $6.1 \pm 0.3^{\prime}$ & $0.39 \pm 0.06$ & $4.2 \pm 0.2^{1}$ & $1.4 \pm 0.1$ & $1.3 \pm 0.2$ \\
\hline$E 4 / 4-3^{* *}$ & 13 & 33.3 & $46.5 \pm 1.9$ & $25.9 \pm 0.9$ & $6.9 \pm 0.3^{\prime}$ & $0.49 \pm 0.05$ & $4.9 \pm 0.3^{\prime}$ & $1.5 \pm 0.1$ & $1.6 \pm 0.1$ \\
\hline
\end{tabular}

* Mean \pm SE. ${ }^{\ddagger}$ Different from E2/2-4 $(P<0.05)$ (Student's $t$ test). 'Different from E3/3 $(P<0.05)$ (Student's $t$ test). "Mean value of the subjects E2/2, E2/3, and E2/4. 'Different from E2/2-4 $(P<0.05$; analysis of variance: $F$ value for total cholesterol is 3.15 and for LDL cholesterol, 4.67.). ** Mean value of the subjects E4/3 and E4/4. HDL, high density lipoprotein. 

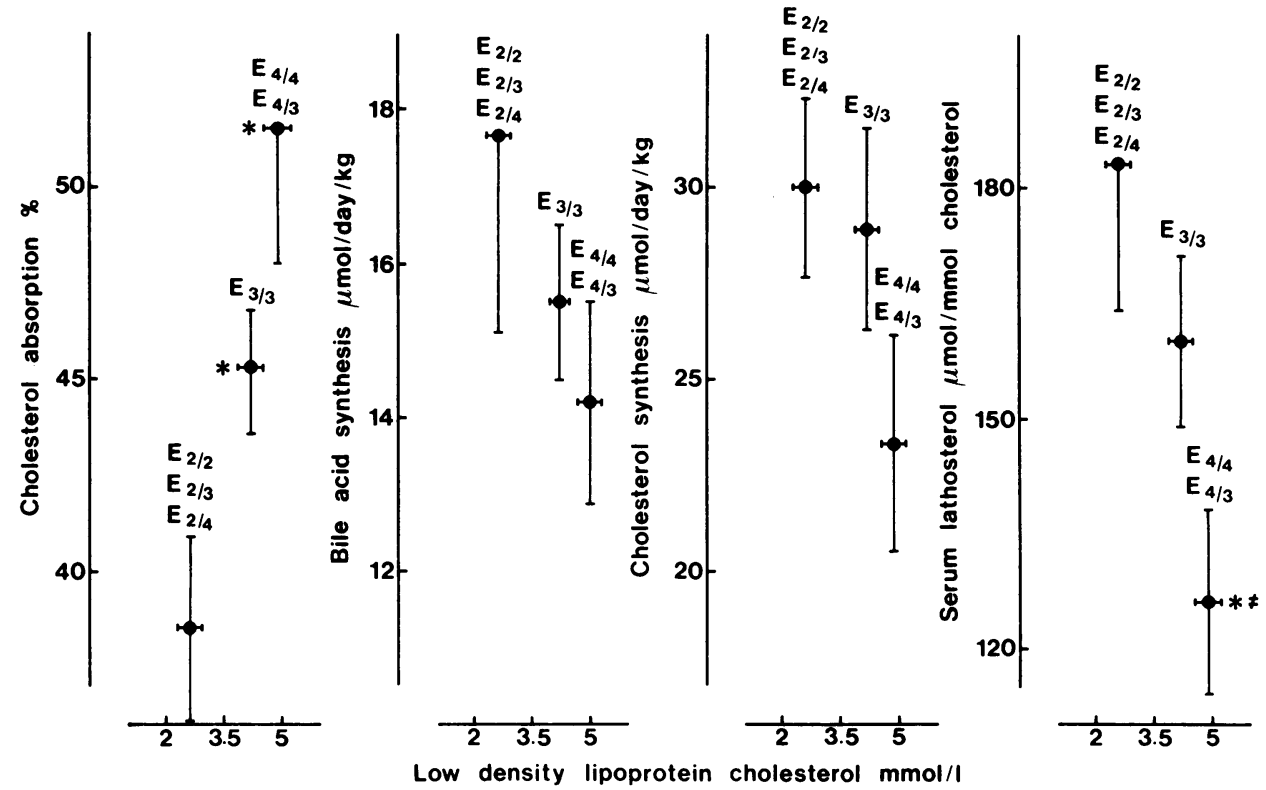

Figure 1. Cholesterol absorption (left), bile acid synthesis (left middle), cholesterol synthesis (right middle), and serum lathosterol (right) in the subjects with various apoE phenotypes. The values are expressed as mean \pm SEM of $4(E 2 / 2, E 2 / 3, E 2 / 4)$, $22(\mathrm{E} 3 / 3)$, and $13(\mathrm{E} 4 / 4, \mathrm{E} 4 / 3)$ subjects. *, Different from the subjects $\mathrm{E} 2 / 2, \mathrm{E} 2 / 3$, and $\mathrm{E} 2 / 4 ; \ddagger$, different from the subject $\mathrm{E} 3 / 3 ;(P<0.05$ or less; analysis of variance; $F$ values of 3.39 for cholesterol absorption and 3.17 for serum lathosterol).

How might apoE regulate the intestinal absorption of cholesterol? The mechanisms can only be a matter of speculation at the moment. The apoE alleles may regulate the transport of cholesterol into the cells, including the hepatocytes, because the hepatic receptor recognition of $\mathrm{apoE}_{2}$-containing lipoproteins is lower than that of apoE3 and apoE4 $(22,23)$. ApoE may also function in the reverse transport of cholesterol from the peripheral cells to the liver for excretion $(27,28)$. ApoE seems to be important in the transport of cholesterol from cholesterol-loaded macrophages (29), and it has been proposed that apoE acts as a shuttle protein to transport cholesterol between various tissues (30). The transport of cholesterol as chylomicrons from the intestinal mucosal cells to the lymph and the liver could be shuttled by mechanisms involving apoE, when apoE polymorphism might also affect this metabolic pathway. ApoE synthesis appears to be negligible in the rat intestinal mucosa $(31,32)$. If the same is true in man, it is difficult to reconcile the present findings with a direct action of apoE at the intestinal mucosal level. If circulating apoE is involved, the shuttling could take place in the mesenteric capillaries as well as in the lymphatics since apoE is also present in lymph. ApoE's regulatory effect on cholesterol absorption efficiency could also be mediated by some unknown secondary messenger, e.g., through a biliary signal from the liver to the intestinal mucosa.

Whatever the mechanism that mediates the association of the apoE phenotype and the efficiency of cholesterol absorption, note that the absorption percentage seems to rise in parallel with the plasma LDL cholesterol level, which, in turn, is related to the apoE phenotype. It can be speculated that this association may disappear if the study population is heterogeneous including, e.g., subjects with familial hypercholesterolemia with completely different mechanisms for high serum cholesterol levels. The presence of subjects with familial-combined hyperlipidemia, other primary hyperlipidemias, or even both genders may similarly distort this relationship.

The observed relationship between the LDL level and cholesterol absorption and the inverse relation between the cholesterol absorption and the activity of cholesterol synthesis in the various apoE phenotypes are in good agreement with the pre- vious findings that the more effectively the hepatocytes are loaded with cholesterol, the less actively the cholesterol is synthesized and the lower is the hepatic LDL (B/E) receptor activity (33, 34). The latter should result in a decreased clearance of LDL from the plasma and subsequently, in elevated LDL levels (33). This was confirmed in the present study. The subjects with homozygosity and heterozygosity for $\epsilon 2$ were poor absorbers of cholesterol, their cholesterol synthesis was high and their plasma LDL levels were low, whereas the $\mathrm{E}_{4}$ patients absorbed cholesterol effectively, had high LDL cholesterol concentrations, and their cholesterol synthesis was low. These findings also indicate that overall cholesterol input (the sum of synthesized plus absorbed dietary cholesterol) may not be associated with the LDL cholesterol concentration, LDL metabolism, or apoE polymorphism because effective absorption reduces cholesterol synthesis. Actually, the calculations on the cholesterol input rates in our patients are in good agreement with the previous work (35), which shows that cholesterol input is not influenced by various apoE isoforms.

Our data would suggest that the LDL receptor activity and clearence of LDL from the plasma are most likely high in the $E_{2}$ subjects and low in the patients with $E 4$. In fact, recent results show that the clearance of $\mathrm{LDL}$ apoB is also related to the apoE phenotype, the subjects with E3/3 having a lower clearance rate than those with E2, but higher than the subjects with E4 (3638). Overall, these data suggest that it may be the variation among the various apoE phenotypes in the absorption of cholesterol and in the uptake of chylomicron remnants that determines the variation of LDL cholesterol concentration associated with the apoE polymorphism.

\section{Acknowledgments}

The authors thank Eeva Gustafsson, Liisa Kuhta, Leena Saikko, Liisa Ikävalko, and Anna-Liisa Launimo for their skilful technical and secretarial assistance.

This study was carried out under a contract with the Finnish Life Insurance Companies and was supported by the Medical Council of the Academy of Finland, the Paavo Nurmi Foundation, and the Sigrid Juselius Foundation. 


\section{References}

1. Utermann, G., I. Kindermann, H. Kaffarnik, and A. Steinmetz. 1984. Apolipoprotein E phenotypes and hyperlipidemia. Hum. Genet. 65:232-236.

2. Assmann, G., G. Schmitz, H.-J. Menzel, and H. Schulte. 1984. Apolipoprotein E polymorphism and hyperlipidemia. Clin. Chem. 30: 641-643.

3. Ehnholm, C., M. Lukka, T. Kuusi, E. Nikkilä, and G. Utermann. 1986. Apolipoprotein E polymorphism in the Finnish population: gene frequencies and relation to lipoprotein concentrations. J. Lipid Res. 27: 227-235.

4. Davignon, J., C. F. Sing, S. Lussier-Cacan, A. C. Nestruck, and D. Bouthillier. 1986. Importance of apolipoprotein E polymorphism in determining plasma lipid levels and atherosclerosis. In Atherosclerosis VII. N. H. Fidge and P. J. Nestel, editors. Elsevier Science Publishers B. V. (Biomedical Div.), Amsterdam. 171-175.

5. Sing, C. F., and Davignon, J. 1985. Role of the apolipoprotein E polymorphism in determining normal plasma lipid and lipoprotein variation. Am. J. Hum. Genet. 37:268-285.

6. Lewis, B., P. R. Turner, J. Revill, R. Konarska, A. LaVille, M. Shaikh, J. Charlton, J. E. Rossouw, M. J. Weight, B. R. Dando, P. L. Jooste, L. Masana, R. Sola, P. Sarda, A. Escobar, J. Salas, M. Mancini, G. Marotta, E. Farinaro, A. Postiglione, Y. A. Kesäniemi, and T. A. Miettinen. 1986. Metabolic epidemiology of plasma cholesterol: mechanisms of variation of plasma cholesterol within populations and between populations. Lancet. II:991-996.

7. Kesäniemi, Y. A., and T. A. Miettinen. 1986. Cholesterol absorption, serum sitosterol and cholesterol synthesis in subjects with low vs. high low density lipoprotein levels. Circulation. 74(Suppl. II): 158.

8. Keys, A. 1970. Coronary heart disease in seven countries. Circulation. XLI(Suppl. 1):I-1-I-19.

9. Ahlström, A., L. Räsänen, and K. Kuvaja. 1972. A method of data processing for food consumption surveys. Ann. Acad. Sci. Fenn. Ser. A IV Biol. 194:1-8.

10. Lipid Research Clinics Manual of Laboratory Operations. 1974. Lipid and Lipoprotein analysis. Department of Health, Education, and Welfare (U.S.) Publication (National Institutes of Health). 1:75-82.

11. Crouse, J., and S. M. Grundy. 1978. Evaluation of continuous isotope feeding method for measurement of cholesterol absorption in man. J. Lipid Res. 19:967-971.

12. Miettinen, T. A., E. H. Ahrens, Jr., and S. M. Grundy. 1965. Quantitative isolation and gas-liquid chromatographic analysis of total dietary and fecal neutral steroids. J. Lipid Res. 6:411-424.

13. Grundy, S. M., E. H. Ahrens, Jr., and T. A. Miettinen. 1965. Quantitative isolation and gas-liquid chromatographic analysis of total fecal bile acids. J. Lipid Res. 6:397-410.

14. Miettinen, T. A. 1982. Gas-liquid chromatographic determination of fecal neutral sterols using a capillary column. Clin. Chim. Acta 124: 245-248.

15. Miettinen, T. A., and P. Koivisto. 1983. Non-cholesterol sterols and bile acid production in hypercholesterolaemic patients with ileal bypass. In Bile Acids and Cholesterol in Health and Disease. G. Paumgartner, A. Stiehl, and W. Gerok, editors. MTP Press Ltd., Lancaster, England. 183-187.

16. Tilvis, R. S., and T. A. Miettinen. 1986. Serum plant sterols and their relation to cholesterol absorption. Am. J. Clin. Nutr. 43:92-97.

17. Miettinen, T. A. 1982. Diurnal variation of cholesterol precursors squalene and methyl sterols in human plasma lipoproteins. J. Lipid Res. 23:466-473.

18. Miettinen, T. A. 1985. Cholesterol precursors and their diurnal rhythm in lipoproteins of patients with jejuno-ileal bypass and ileal dysfunction. Metabolism. 34:425-430.

19. Utermann, G., M. Jaeschke, and J. Menzel. 1975. Familial hyperlipoproteinemia type III: deficiency of a specific apolipoprotein (apoEIII) in the very low density lipoproteins. FEBS (Fed. Eur. Biochem. Soc.) Lett. 56:325-355.
20. Zannis, V. I., and J. L. Breslow. 1981. Human very low density lipoprotein apolipoprotein $\mathrm{E}$ isoprotein polymorphism is explained by genetic variation and post-translational modification. Biochemistry. 20: 1033-1041.

21. Rall, S. C., Jr., K. H. Weisgraber, and R. W. Mahley. 1982. Human apolipoprotein E: the complete amino acid sequence. J. Biol. Chem. 257:4171-4178.

22. Weisgraber, K. H., T. L. Innerarity, and R. W. Mahley. 1982. Abnormal lipoprotein receptor-binding activity of the human $\mathrm{E}$ apoprotein due to cysteine-arginine interchange at a single site. J. Biol. Chem. 257:2518-2521.

23. Mahley, R. W., and T. L. Innerarity. 1983. Lipoprotein receptors and cholesterol homeostasis. Biochim. Biophys. Acta. 737:197-222.

24. Gregg, R. E., L. A. Zech, E. J. Schaefer, and H. B. Brewer, Jr. 1981. Type III hyperlipoproteinemia: defective metabolism of an abnormal apolipoprotein E. Science (Wash. DC). 211:584-586.

25. Stalenhoef, A. F. H., M. J. Malloy, J. P. Kane, and R. J. Havel. 1986. Metabolism of apolipoproteins B-48 and B-100 of triglyceriderich lipoproteins in patients with familial dysbetalipoproteinemia. J. Clin. Invest. 78:722-728.

26. Gregg, R. E., L. A. Zech, E. J. Schaefer, D. Stark, D. Wilson, and H. B. Brewer, Jr. 1986. Abnormal in vivo metabolism of apolipoprotein $\mathrm{E}_{4}$ in humans. J. Clin. Invest. 78:815-821.

27. Glomset, J. A. 1968. The plasma lecithin: cholesterol acyltransferase reaction. J. Lipid Res. 9:155-167.

28. Koo, C., T. L. Innerarity, and R. W. Mahley. 1985. Obligatory role of cholesterol and apolipoprotein $\mathrm{E}$ in the formation of large cholesterol-enriched and receptor-active high density lipoproteins. J. Biol. Chem. 260:11934-11943.

29. Basu, S. K., M. S. Brown, Y. K. Ho, R. J. Havel, and J. L. Goldstein. 1981. Mouse magrophages synthesize and secrete a protein resembling apolipoprotein E. Proc. Natl. Acad. Sci. USA. 78:7545-7549.

30. Blue, M.-L., D. L. Williams, S. Zucker, S. A. Chan, and C. B. Blum. 1983. Apolipoprotein E synthesis in human kidney, adrenal gland and liver. Proc. Natl. Acad. Sci. USA. 80:283-287.

31. Elshourbagy, N. A., W. S. Liao, R. W. Mahley, and J. M. Taylor. 1985. Apolipoprotein E mRNA is abundant in the brain and adrenals, as well as in the liver, and is present in other peripheral tissues of rats and marmosets. Proc. Natl. Acad. Sci. USA. 82:203-207.

32. Driscoll, D. M., and G. S. Getz. 1984. Extrahepatic synthesis of apolipoprotein E. J. Lipid Res. 25:1368-1379.

33. Brown, M. S., and J. L. Goldstein. 1983. Lipoprotein receptors in the liver. Control signals for plasma cholesterol traffic. J. Clin. Invest. 72:743-747.

34. Angelin, B., C. A. Raviola, T. L. Innerarity, and R. W. Mahley. 1983. Regulation of hepatic lipoprotein receptors in the dog. Rapid regulation of apolipoprotein B, E receptors, but not of apolipoprotein E receptors, by intestinal lipoproteins and bile acids. J. Clin. Invest. 71: 816-831.

35. Palmer, R. H., A. V. Nichols, R. B. Dell, R. Ramakrishnan, F. T. Lindgren, E. L. Gong, C. R. Blum, and D. S. Goodman. 1986. Lack of relationship in humans of the parameters of body cholesterol metabolism with plasma levels of subfractions of HDL or LDL, or with apoE isoform phenotype. J. Lipid Res. 27:637-644.

36. Gregg, R. E., and M. B. Brewer, Jr. 1986. Modulation of the metabolism of apolipoprotein B-48 and apolipoprotein B-100 containing lipoproteins by apolipoprotein E. In Atherosclerosis VII. N. H. Fidge and P. J. Nestel, editors. Elsevier Science Publishers B. V. (Biomedical Div.), Amsterdam. 335-339.

37. Kesäniemi, Y. A., C. Ehnholm, and T. A. Miettinen. 1986. Apoprotein E phenotype's powerful role in low density lipoprotein catabolism. Arteriosclerosis. 6:541. (Abstr.)

38. Kesäniemi, Y. A., M. Färkkilä, K. Kervinen, P. Koivisto, M. Vuoristo, and T. A. Miettinen. 1987. Regulation of low density lipoprotein apolipoprotein B levels. Am. Heart J. 113:508-513. 